\title{
Valor energético del aceite usado en sistemas de combustión térmicos de la industria cementera del país
}

\section{Energy value of oil used in thermal combustion systems of the cementing industry of the country}

Rafael Santiago Albuja Echeverría. ${ }^{1}$, Julio César López Ayala. ${ }^{2}$, Jaime Rodrigo Guilcapi Mosquera. ${ }^{3}$ \& Carlos Horacio Guamán Reinoso. ${ }^{4}$

Recibido: 1-10-2019 / Revisado: 25-10-2019 /Aceptado: 04-11-2019/ Publicado: 07-12-2019

\begin{abstract}
.
DOI https://doi.org/10.33262/cienciadigital.v3i4.2.1012
\end{abstract}

The replacement of used lubricating oils generated by automotive sources, as part of the fuel handled in cement kilns; It is a strategy that improves procedures before a final disposal of this type of highly polluting hazardous waste. The study statistically checks the characteristics of the used lubricating oils generated by the automotive park of the city of Riobamba, determining that its physical-chemical composition is suitable for an energy recovery, by using the oven fuel injection system as a percentage cement maker. It is estimated that around 63-64 $\mathrm{t} /$ year of oily bases used in Ecuador enter various sectors of the national economy, obtaining a collection of used lubricating oil of 61736 $\mathrm{kg} /$ year, where $16 \%$ are treated by authorized managers, what represents an inadequate technological and environmental management. The objective of this

1 Escuela Superior Politécnica de Chimborazo, Facultad de Mecánica, Riobamba, Ecuador., ralbuja@espoch.edu.ec

2 Universidad Nacional de Chimborazo, Facultad de Ingeniería, Carrera Industrial, Riobamba, Ecuador., 1982jcla@gmail.com

3 Universidad Técnica de Ambato, FISEI, Carrera de Telecomunicaciones, Ambato, Ecuador, jr.guilcapi@uta.edu,ec

4 Hospital General Docente de Ambato, Ecuador, calosho11@yahoo.com 
research is to assess the characteristics of the energy potential of this hazardous waste for use as fuel in cement kilns, for which 12 mixtures were analyzed combined between used oil and a fuel of fossil origin collected by the industry and automotive sources from the city of Riobamba; the heat capacity for fuels is determined, comparing with what is required under international regulations. The analysis of mixtures was treated in a statistical way under the method of ANOVA and DUNNET comparison, which have a high calorific value, superior to $9300 \mathrm{kcal} / \mathrm{kg}$, and an optimum characteristic for energy use for thermal combustion systems in the cement industry. The results show that the lubricating oils used are suitable for the use of fuel, due to their heat capacity; becoming an alternative for the solution to the problem of final disposal of hazardous waste of the city and environmental protection regulated under the environmental management law of the country.

Keywords: Used lubricating oil, cement industry, statistical method ANOVA and DUNNET,

\section{Resumen.}

Se estima que en Ecuador ingresan alrededor de $63-64 \mathrm{t}$ /año de bases aceitosas utilizados en diversos sectores de la economía nacional, obteniéndose una recolección de aceite lubricante usado de $61736 \mathrm{~kg} /$ año, donde el 16\% son tratados por gestores autorizados, lo que representa una inadecuada gestión tecnológica y ambiental. El objetivo de esta investigación es valorar las características del potencial energético de este desecho peligroso para su uso como combustible en hornos cementeros, para lo cual se analizó 12 mezclas combinadas entre el aceite usado y un combustible de origen fósil recolectado por la industria y fuentes automotrices de la ciudad de Riobamba; se determina la capacidad calorífica para combustibles, comparándose con lo exigido bajo normativa internacional. El análisis de mezclas fue tratado de forma estadística bajo el método de ANOVA y comparación DUNNET, las mismas que poseen un elevado poder calorífico, superior a $9300 \mathrm{kcal} / \mathrm{kg}$, característica óptima al aprovechamiento energético para los sistemas térmicos de combustión del sector industrial cementero. Los resultados demuestran que los aceites lubricantes usados son aptos para la utilización de combustible, por su capacidad calorífica; convirtiéndose en alternativa para la solución al problema de disposición final de residuos peligrosos de la ciudad y protección del medio ambiente regulados bajo la ley de gestión ambiental del país.

Palabras claves: Aceite lubricante usado, industria cementera, método estadístico ANOVA y DUNNET 


\section{Introducción.}

La demanda mundial de aceites lubricantes es de aproximadamente 44 millones de toneladas al año, con un crecimiento a largo plazo del $6 \%$ conforme al crecimiento industrial y automotor. (Lubrication \&Management, 2016).

En el Ecuador en el año 2012 según datos reportados del Banco Central la demanda comercial fue aproximadamente 63497 toneladas, la Figura 1 muestra la cantidad de residuos recolectados, para el caso de los aceites usados es de 61 736,76 kg/año; el 56\% de los desechos peligrosos no reciben ningún tipo de tratamiento, el 32\% se sujetan a algún tipo de reciclaje, el 12\% reciben algún tipo de tratamiento previo a su descarga. (INEC, 2012). Actualmente la valorización energética de los aceites lubricantes usados es reutilizada en procesos de combustión industrial acorde avances tecnológicos existentes; aprovechándolos ya sea como materia prima o como energéticos de forma segura para la protección del medio ambiente. (Gonzales, 2008).

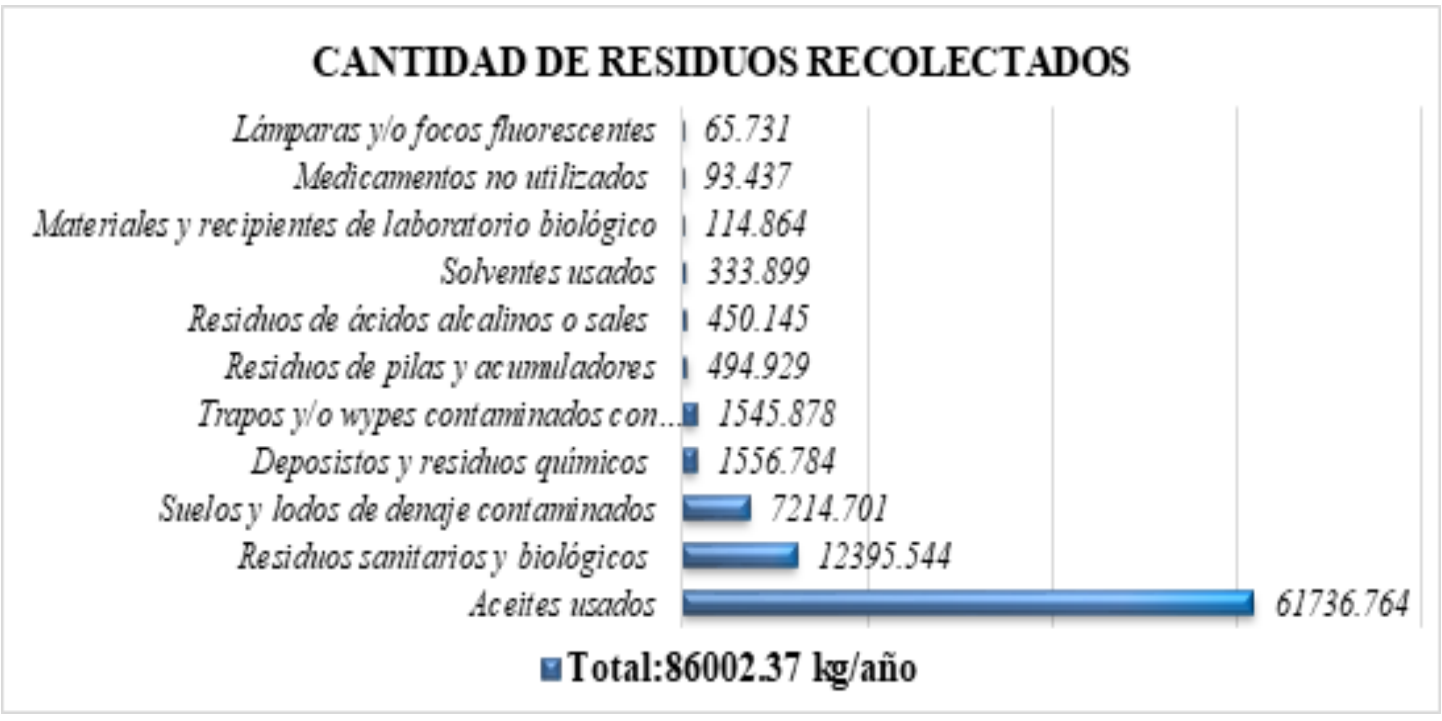

Figura 1: Cantidad de residuos peligrosos registrados al año.

Fuente: Instituto Nacional de Estadísticas y Censos INEC, 2012

En el Ecuador no existe un control adecuado del aceite lubricante usado, la liberación de partículas contaminantes toxicas son consideradas potencialmente peligrosas para el medio ambiente. Se estima una generación anual estimada de 86002 toneladas de desechos peligrosos, el $44.5 \%$ se encuentran en estado líquido, el $55 \%$ en estado sólido y el $0.5 \%$ en estado gaseoso. (Gordon, 2005).

En cuanto a la salud y seguridad ambiental, la autoridad sanitaria nacional en coordinación con el Ministerio de Medio Ambiente, establece normas básicas para la preservación del 
ambiente. La gestión de desechos peligrosos define al desecho peligroso a aquellos desechos sólidos, pastosos, líquidos o gaseosos resultantes de un proceso de producción, transformación, reciclaje, utilización o consumo y que contengan algún compuesto que tenga características reactivas, inflamables, corrosivas, infecciosas o tóxicas, que represente un riesgo para la salud humana, los recursos naturales y el ambiente de acuerdo a las disposiciones legales. (TULS, 2015).

En los últimos años han surgido nuevos procesos y tecnologías que permiten la reutilización o reciclaje de los aceites usados, transformándolos en productos susceptibles de ser utilizados para aprovechamiento energético por su elevada capacidad calorífica, convirtiéndolos en uno de los residuos con mayor potencial como combustible Los aceites lubricantes usados conservan grandes cantidades de energía interna que son utilizados por el sector industrial cementero, como parte del proceso de combustión en hornos de producción de clínker, siempre que sus características lo permitan. (Delgado \& Parra 2007)

Para el caso de los aceites lubricantes usados, pueden tener impactos importantes tanto en la salud de las personas como en el medio ambiente, al caracterizarlo de manera correcta al proceso de combustión en hornos de procesamiento de clínker al proceso de fabricación del cemento no existe un incremento de emisiones sino un ahorro relevante en términos de emisiones de CO2, NOx y SO2 liberadas a la atmosfera. (Genon 2008).

La caracterización del poder calorífico del aceite lubricante usado generado por la industria y el parque automotor de la ciudad de Riobamba, valora las características de este desecho para su uso como combustible, único destino final la combustión en un horno de clínker.

En la investigación se estudian doce mezclas de aceites usados recolectados en diversas fuentes de contaminación de la ciudad; se determinan la capacidad calorífica dentro de sus propiedades físicas y químicas fundamentales para combustibles, y se comparan con requisitos establecidos en especificaciones internacionales. (López, 2016).

Los combustibles alternos, el análisis por (Diosdado, 2009), menciona que los residuos industriales más adecuados para la formulación de combustibles alternos son los aceites y grasas usados, solventes gastados, lodos de pinturas, lodos orgánicos, adhesivos, residuos plásticos, entre otros. Esta alternativa permite seleccionar el componente del aceite usado con cualquier otro combustible de origen fósil generándose en variable investigativa y desarrollo tecnológico, que permitan el aprovechamiento energético de estos recursos a partir de criterios de alternativas de gestión tecnológica ambiental.

Dentro del poder calorífico de combustión para la industria cementera según la norma ASTM D 240, menciona "es la cantidad de energía liberada cuando se quema una masa unitaria de combustible a presión constante, siendo todos los productos, incluyendo el agua, gaseosos". (American Society for Testing and Materials, 1997). Los resultados son reportados en kilocalorías sobre kilogramo; la ecuación 1 utilizo la transformación de las unidades. 
Por su capacidad calorífica el aceite lubricante usado se constituye en uno de los residuos con mayor potencial a ser empleado como combustible en los hornos cementeros. El valor generado por el poder calorífico depende del análisis que precisan al cálculo de un sistema de combustión. Las características que hacen de los hornos de cemento una alternativa técnica y ambientalmente adecuada para el tratamiento de residuos lo explica el a partir de los siguientes dos aspectos: la destrucción de componentes orgánicos, la recuperación de energía y reducción de emisiones a la atmósfera. (GTZ/Holcim, 2006).

La definición en base la caracterización del poder calorífico del aceite usado se realizó un tratamiento estadístico con el método ANOVA y comparación por DUNNET. Los resultados evidenciaron que la caracterización de varias mezclas entre un combustible de origen fósil y el aceite lubricante usado, alcanza un poder calorífico optimo, demostrándose que no existe diferencias significativas a la variable analizada. La tecnología de procesamiento, es el grado de sustitución de los combustibles convencionales por combustibles alternos en un horno de clínker depende de la composición y el poder calorífico.

El coprocesamiento en la industria cementera es la forma óptima de recuperación de la energía y la materia de residuos.

Ofrece una solución sólida y segura para la sociedad, el medio ambiente y la industria cementera, sustituyendo los recursos no renovables por residuos bajo estrictas medidas de control.

La recuperación de residuos en la industria cementera entre ellos los aceites usados, también llamada coprocesado, contribuye a facilitar la posibilidad de alcanzar un máximo de sustitución de materiales no renovables. Los tipos de residuos que pueden usarse en una planta cementera varían en función de cada instalación. Como regla básica, los residuos aceptados como combustible y/o materia prima alternativa deben aportar un valor añadido al horno de cemento en términos de poder calorífico del valor material de la parte mineral. Algunos combustibles alternativos cumplirán a la vez ambos requisitos, haciendo difícil formular un criterio general en relación a los materiales que son coprocesados en la industria cementera.

Según las características del proceso de producción, la industria cementera puede coprocesar, combustibles alternativos que tengan un importante poder calorífico para el caso de aceites usados. Es estrictamente necesario que exista un correcto sistema de control de calidad para todos los materiales utilizados, ya que, de esta forma, se asegura que el coprocesado se lleva a cabo de una manera medioambientalmente segura, manteniendo:

- La seguridad y salud de la población.

- El comportamiento medioambiental del proceso de producción.

- La alta calidad del producto final. 
Un proceso de producción correcto e ininterrumpido.La combustión en los hornos de cemento se realiza con un exceso de oxígeno que debe limitarse para no sacrificar en exceso la eficiencia energética que se encuentra condicionada, además, a la uniformidad del combustible y a su adecuado manejo para facilitar una correcta y completa combustión. Por su capacidad calorífica el aceite usado se constituye en uno de los residuos con mayor potencial a ser empleado como combustible en los hornos cementeros. Es factible estimar no solo por sus propiedades y características sino por la necesidad de contar con alternativas energéticas en la industria cementera.

\section{Metodología}

La modalidad básica de la investigación es de campo, ya que las variables objeto a estudio son recolectadas en el lugar y tiempo en que ocurren los fenómenos objeto de estudio (Grajales, 2017); de laboratorio, puesto que las muestras son analizadas para obtener el poder calorífico de cada mezcla; bibliográfico documental para conocer las cantidades de aceite lubricante usado en la ciudad de Riobamba.

La metodología de esta investigación parte de la norma internacional ASTM D 4057:2009; método de prueba estándar para muestreo manual de productos derivados del petróleo. Los resultados de la caracterización del poder calorífico neto en función a las mezclas de los índices físico -químicos se la realizaron bajo ensayo ASTM D 6448 y D 6823, indicadas en la Tabla 1; siendo para la presente investigación el analizado dentro del grado RFO4.

Tabla 1: Índices de calidad de combustibles a partir de aceites usados

\begin{tabular}{|c|c|c|c|c|}
\hline \multirow[t]{2}{*}{ Índices de calidad Método } & \multicolumn{4}{|l|}{ Grados } \\
\hline & $\overline{\mathrm{RFO} 4}$ & RFO5L & RFO5H & RFO6 \\
\hline $\begin{array}{l}\text { Valor calórico ASTM I } \\
\text { neto, } \mathrm{kcal} / \mathrm{kg}\end{array}$ & 9300 mín. & 9920 mín & 9920 mír & 10 140mín. \\
\hline
\end{tabular}

Esta especificación cubre cuatro calidades de combustible fabricado en su totalidad o en parte con aceite lubricante usado o reprocesado a base de hidrocarburos o fluidos funcionales. Los cuatro grados de combustible están diseñados para ser utilizados en diversos tipos de equipos industriales que queman combustibles en diversas condiciones climáticas y de operación.

Los grados RFO4, RFO5L, RFO5H y RFO6 se usan mezclas de aceite lubricante, con o sin destilado o fuel oil residual, o ambos, de viscosidad creciente y están diseñados para 
usarse en quemadores industriales equipados para manejar estos tipos de combustibles reciclados

Nota: Esta especificación no deberá impedir el cumplimiento de las regulaciones nacionales o locales, que pueden ser más restrictivas. En algunas jurisdicciones, el aceite usado se considera un residuo peligroso y los combustibles del aceite usado deben cumplir ciertos criterios antes de su uso como combustible. Los valores son los mínimos requeridos.

\section{Resultados}

\section{Modelación y procesamiento de resultados}

El análisis físico químico del poder calorífico inició con el procesamiento de los resul obtenidos de las muestras tomadas entre el aceite lubricante usado generado por el pi automotor y la industria cemento con mayor capacidad productiva y económica de la ciudi Riobamba.

Para lo cual la frontera del estudio del poder calorífico analizó la composición porcentual en aceite lubricante usado y un combustible de origen fósil siendo este el residuo petrolero indu utilizado en hornos de procesamiento de clínker, materia prima principal al proceso de fabrici del cemento.

El análisis caracterizó, distintas mezclas recolectadas bajo técnica de sustitución porcentu información expuesta en la Tabla 3 ilustra el porcentaje de muestras experimentadas combustible de origen fósil y aceite lubricante usado. El tratamiento corresponde a un cr designado por muestra (MA, MB, MC, MD) que identifica para cada uno un total de doce prı ensayadas con cantidades de forma porcentual por un laboratorio certificado.

Tabla 3: Porcentaje de muestras experimentadas

\begin{tabular}{llll}
\hline Combustible industrial & Aceite lubricante usado & Muestra & Total pruebas \\
\hline $90 \%$ & $10 \%$ & MA & PA1, PA3, PA9 \\
$80 \%$ & $20 \%$ & MB & PB2, PB4, PB6 \\
$70 \%$ & $30 \%$ & MC & PC5, PC7, PC11 \\
$60 \%$ & $40 \%$ & MD & PD8, PD10, PD12 \\
\hline
\end{tabular}

Realizado por: Julio López, 2018 
Muestra MA. $90 \mathrm{~cm}^{3}$ Combustible Industrial $+10 \mathrm{~cm}^{3}$ de Aceite Lubricante Usado en un litro de Combustible analizado. Total, de Pruebas ensayadas PA1-PA3-PA9.

Muestra MB. $80 \mathrm{~cm}^{3}$ Combustible Industrial $+20 \mathrm{~cm}^{3}$ de Aceite Lubricante Usado en un litro de Combustible analizado. Total, de Pruebas ensayadas PB2-PB4-PB6.

Muestra MC. $70 \mathrm{~cm}^{3}$ Combustible Industrial $+30 \mathrm{~cm}^{3}$ de Aceite Lubricante Usado en un litro de Combustible analizado. Total, de Pruebas ensayadas PC5-PC7-PC11.

Muestra MD. $60 \mathrm{~cm}^{3}$ Combustible Industrial $+40 \mathrm{~cm}^{3}$ de Aceite Lubricante Usado en un litro de Combustible analizado. Total, de Pruebas ensayadas PD8-PD10-PD12.

El procesamiento de resultados inicia con la modelación estadística del análisis de varianza, para lo cual se utiliza un grupo de control llamado tratamiento, para lo cual se tabuló de forma estadística la variable del poder calorífico entre la mezcla del aceite lubricante usado y combustible industrial, comprobándose si presenta alguna diferencia significativa en cuanto a la variable tratada. Para lo cual verificó el rechazo de la hipótesis nula al control de los tratamientos bajo la comparación múltiple y posterior a ello confirmándose con la prueba de DUNNET.

\section{Resultados del poder calorífico de mezclas}

Los resultados del poder calorífico la composición porcentual de las muestras analizadas MA, MB, MC, MD doce muestras entre el aceite lubricante usado y un combustible industrial del sector cementero emitidos por el laboratorio de la Facultad de Ingeniería Química de la Universidad Central del Ecuador bajo la norma ASTM D 240; son presentados en la Tabla 4.

Tabla 4: Resultados del poder calorífico del aceite usado y combustible industrial

\begin{tabular}{|c|c|c|c|c|}
\hline Resultados mezcla & \multicolumn{4}{|c|}{$90 \%$ Combustible industrial } \\
\hline \multirow[t]{4}{*}{ MA } & \multicolumn{4}{|l|}{$10 \%$ Aceite usado } \\
\hline & \multirow{3}{*}{$\begin{array}{l}\text { Poder Calorífico } \\
\text { Bruto (kcal/kg) }\end{array}$} & \multicolumn{3}{|c|}{ Muestras } \\
\hline & & $\mathrm{P} 1$ & P5 & P9 \\
\hline & & 8608 & 9545 & 9304 \\
\hline Resultados mezcla & \multicolumn{4}{|c|}{$80 \%$ Residuo industrial } \\
\hline \multirow[t]{4}{*}{$\mathrm{MB}$} & \multicolumn{4}{|l|}{$20 \%$ Aceite usado } \\
\hline & \multirow{3}{*}{$\begin{array}{l}\text { Poder Calorífico } \\
\text { Bruto (kcal/kg) }\end{array}$} & \multicolumn{3}{|c|}{ Muestras } \\
\hline & & $\mathrm{P} 2$ & P6 & $\mathrm{P} 10$ \\
\hline & & 9503 & 9283 & 9166 \\
\hline Resultados mezcla & \multicolumn{4}{|c|}{$70 \%$ Residuo Industrial } \\
\hline $\mathrm{MC}$ & \multicolumn{4}{|l|}{$30 \%$ Aceite Usado } \\
\hline & $\begin{array}{l}\text { Poder Calorífico } \\
\text { Bruto }(\mathrm{kcal} / \mathrm{kg})\end{array}$ & \multicolumn{3}{|c|}{ Muestras } \\
\hline
\end{tabular}




\begin{tabular}{|c|c|c|c|c|}
\hline & & P3 & P7 & $\mathrm{P} 11$ \\
\hline & & 9564 & 9561 & 9184 \\
\hline Resultados mezcla & \multicolumn{4}{|c|}{$60 \%$ Residuo Industrial } \\
\hline \multirow[t]{4}{*}{$\mathrm{MD}$} & \multicolumn{4}{|l|}{$40 \%$ Aceite Usado } \\
\hline & \multirow{3}{*}{$\begin{array}{l}\text { Poder Calorífico } \\
\text { Bruto (kcal/kg) }\end{array}$} & \multicolumn{3}{|c|}{ Muestras } \\
\hline & & $\mathrm{P} 4$ & P8 & P12 \\
\hline & & 9338 & 9566 & 9122 \\
\hline
\end{tabular}

Fuente: Resultados laboratorio de la UCE Escuela de Ingeniería Química, 2016

\section{Demostración experimental}

Los resultados del poder calorífico expuestos en la Tabla 4, proporcionaron la demostración experimental de las distintas mezclas entre el combustible industrial y el aceite lubricante usado donde sus resultados evaluaron mediante un tratamiento estadístico la mezcla óptima al proceso de combustión para sistemas térmicos de un horno cementero.

\begin{tabular}{|c|c|c|c|c|c|}
\hline \multirow[t]{4}{*}{ Observaciones } & \multirow[t]{4}{*}{$y i j$} & A & B & $\mathrm{C}$ & D \\
\hline & & 8608 & 9503 & 9564 & 9338 \\
\hline & & 9545 & 9283 & 9561 & 9566 \\
\hline & & 9304 & 9166 & 9184 & 9122 \\
\hline \multirow[t]{5}{*}{ Sumatoria } & & 27457 & 27952 & 28309 & 28026 \\
\hline & $\begin{array}{lll}y & i\end{array}$ & 9152,33 & 9317,33 & 9436,33 & 9342,00 \\
\hline & $\hat{y}$ & $\hat{y}_{1}$ & $\hat{y}_{2}$ & $\hat{y}_{3}$ & $\hat{y}_{4}$ \\
\hline & $n=$ & \multicolumn{4}{|c|}{3} \\
\hline & $k=$ & \multicolumn{4}{|c|}{4} \\
\hline
\end{tabular}

Figura 3: Evaluación de resultados al tratamiento estadístico en base al análisis de varianza ANOVA.

Fuente: Julio López, 2018

La Figura 4 y 5, corresponde a la evaluación de resultados al tratamiento estadístico en base al análisis de la varianza ANOVA, demostración que utilizó un Procedimiento analítico y herramientas informáticas de Microsoft Excel. 


\section{PODER CALORÍFICO ( $\mathrm{kcal} / \mathrm{kg})$}

\section{Hipótesis:}

$\mathrm{H}_{0}=\mu_{1}=\mu_{2}$

$\mathrm{H}_{1}=\mathrm{Al}$ menos 2 de las medidas no son iguales

\section{Método analítico}

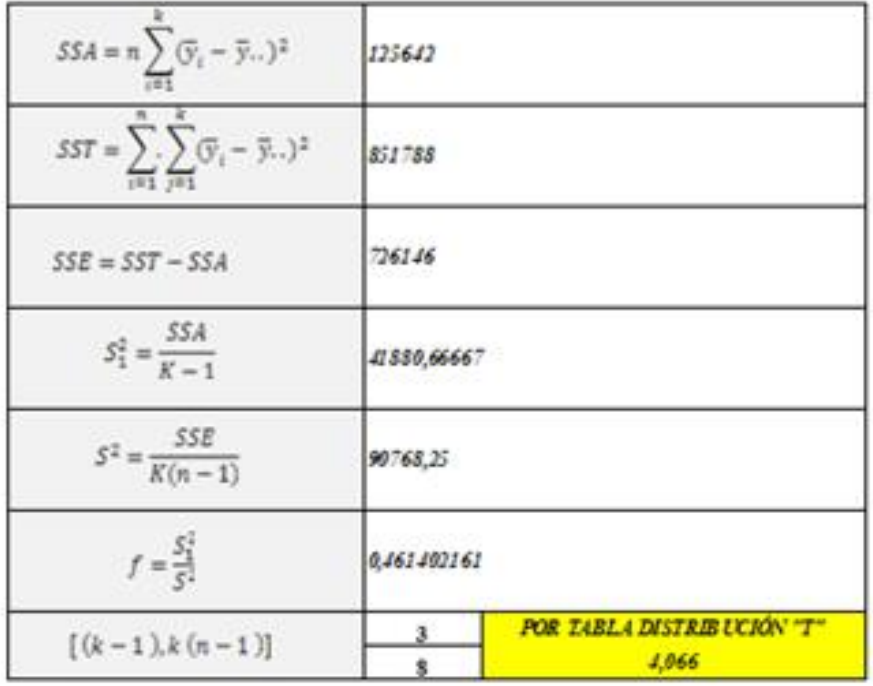

\section{Hoja de cálculo Microsoft Excel}

\begin{tabular}{|l|l|l|l|l|l|l|}
\hline $\begin{array}{l}\text { Origen de las } \\
\text { variaciones }\end{array}$ & $\begin{array}{c}\text { Suma de } \\
\text { cuadrados }\end{array}$ & $\begin{array}{c}\text { Grados de } \\
\text { libertad }\end{array}$ & $\begin{array}{c}\text { Promedio de } \\
\text { los cuadrados }\end{array}$ & F & Probabilidad & $\begin{array}{c}\text { Valor critico } \\
\text { para F }\end{array}$ \\
\hline Entre grupos & 125642 & 3 & 41880,66667 & 0,4614 & 0,716935425 & 4,066180551 \\
\hline Dentro de los grupos & 726146 & 8 & 90768,25 & & & \\
\hline Total & 851788 & 11 & & & & \\
\hline
\end{tabular}

Figura 4: Análisis del poder calorífico método ANOVA

Fuente: Julio López, 2018

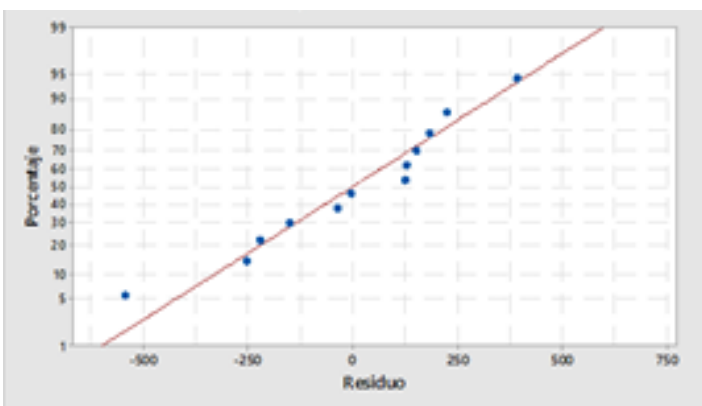

Figura 5: Probabilidad normal de muestras MA-MB-MC-MD

Fuente: Julio López, 2018 
La Figura 6, corresponden a la evaluación de resultados y comprobación con la prueba de DUNNET, cuya hipótesis establece la existencia de diferencias significativas a la variable del poder calorífico que pudieran afectar al proceso de combustión, demostración que utilizó la herramienta informática versión 1.7 de Minitab.

\section{PODER CALORIFICO (kcal/kg)}

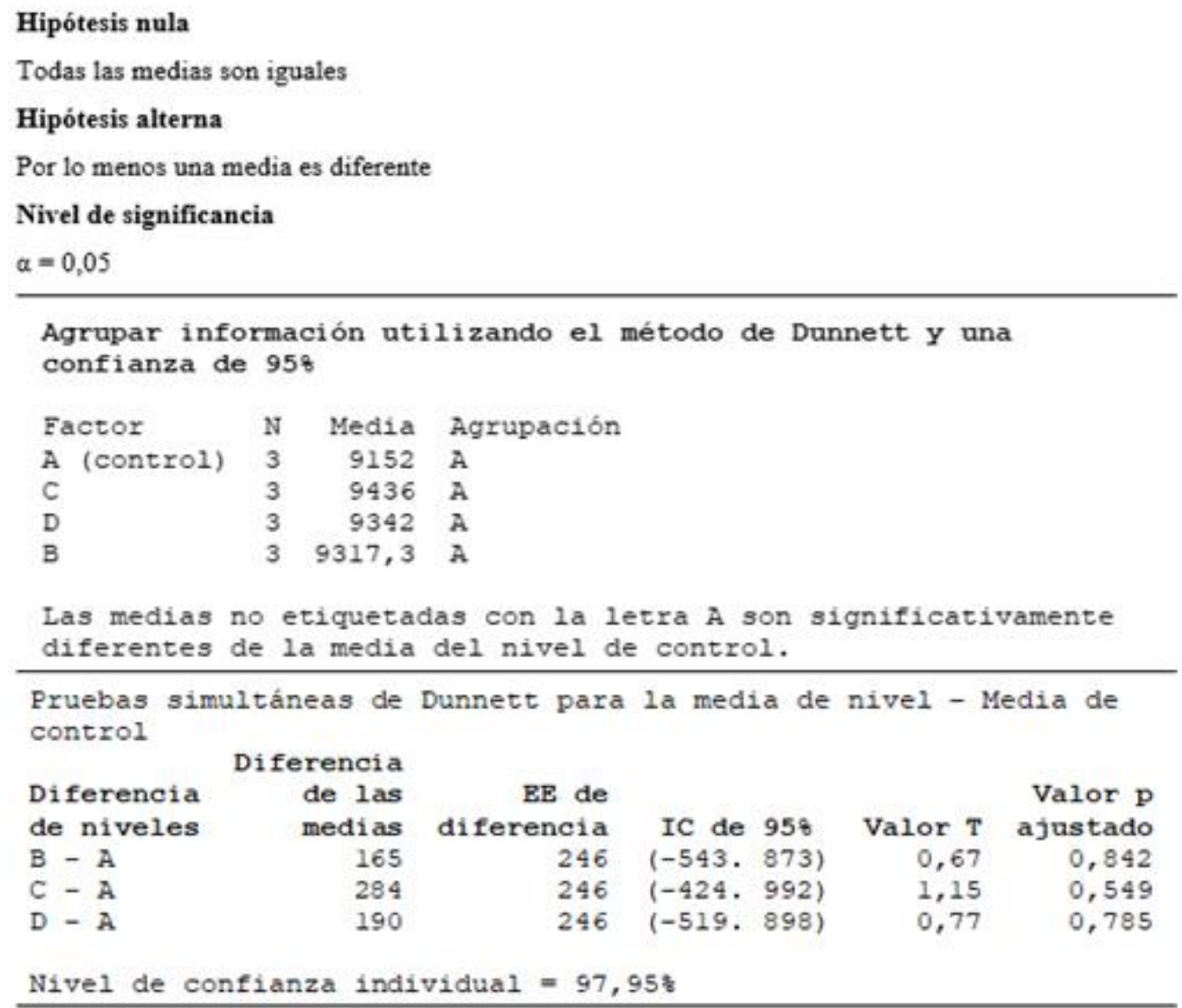

Grófico Dunnett: A. B. C. D

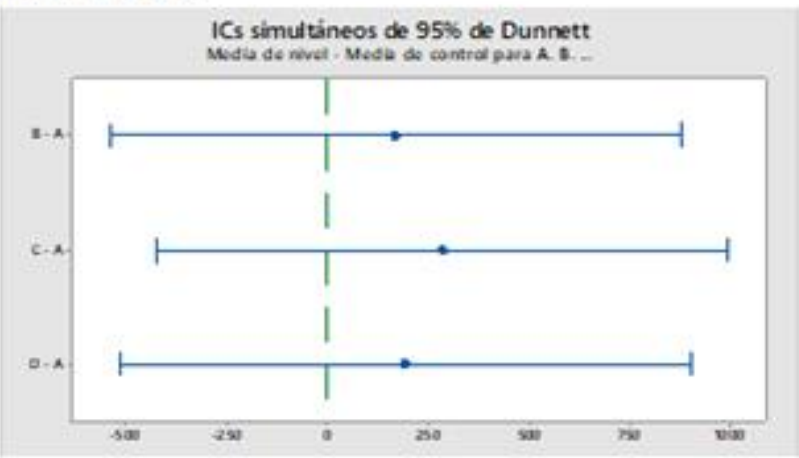

Figura 6: Análisis del poder calorífico comprobación método DUNNET

Fuente: Julio López, 2018 
Los resultados analizados de la modelación estadística de ANOVA y comprobación múltiple por DUNNET, demostró que la Hipótesis nula se acepta, el poder calorífico obtenido de los tratamientos no existe diferencias significativas en sus variables en cada una de las mezclas.

\section{Valorización energética análisis del costo \& beneficio}

El coprocesamiento de aceites usados genera un valor tanto desde punto de vista ambiental y energético; y, su valor económico lo determina el mercado informal.

El análisis productivo del horno cementero tiene un promedio de 600 toneladas de clínker al día, para producir una tonelada se utiliza en un promedio 26 galones de residuo industrial petrolero a un valor de $0.64 \mathrm{USD} /$ galón. El análisis costo beneficio se observa de acuerdo a una con un costos de 0.34 USD/galón, demanda que analiza una valorización energética del aceite lubricante usado observado en la figura 7, correspondiente al análisis de costo \& beneficio por coprocesamiento de aceite usados.

\begin{tabular}{llll}
\hline \multicolumn{4}{c}{ PROYECCIÓN DE AHORRO POR COSTOS DE ENERGÍA TÉRMICA } \\
\hline \multirow{2}{*}{ AÑO } & Generación anual & Costo anual & Costos anuales \\
& Aceites usados $(\mathrm{gls})$ & $(0.64$ USD) & $(0.34$ USD) \\
& & Residuo Petrolero & Aceite usados \\
\hline 2017 & 316044 & $\$ 202.268,16$ & $\$ 107.454,96$ \\
\hline \multirow{4}{*}{ AHORRO ENERGÉ- } & \$ 94.813,20 USD & \\
& TICO ANUAL & & \\
\hline
\end{tabular}

Figura 7: Análisis de costo \& beneficio por procesamiento de aceite usados.

Realzado por: Julio López, 2018

En general el aceite usado posee ventajas energéticas como su elevado poder calorífico, y no necesita un tratamiento de calentamiento para su inyección en el horno, por ende, existe un ahorro de energía. El programa de recolección y disposición final de los aceites usados frente a una gestión integral con adecuadas prácticas ambientales, conllevó a realizar encuestas a 30 empresas que generan aceites lubricantes usados en la ciudad de Riobamba, dichas empresas fueron 19 mecánicas, 5 Lavadoras, 6 Lubricadoras, obteniéndose resultados observados en la figura 7 cabe señalar que este análisis admitió tener pérdidas en la extracción de los mismos, así no cuentan con respaldos físicos que validen la cantidad exacta del volumen que se maneja dichas instalaciones. Además, se evidenció además que el $96,67 \%$ no posee una zona delimitada para el almacenamiento seguro de los aceites extraídos de los vehículos. 


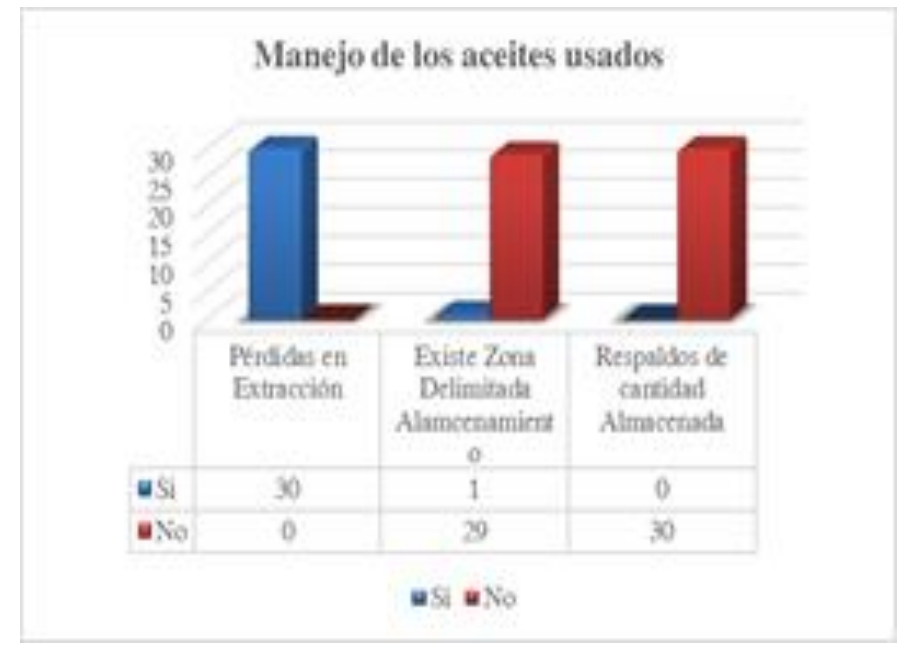

Figura 7: Condiciones de manejo de aceites usados

Realzado por: Julio López, 2018

\section{Conclusiones.}

- El ahorro energético se proyectó en base al costo beneficio respecto al precio de residuo petrolero al año 2018, el mismo que estaría cerca de 58.488,67 USD de energía térmica; La potencialidad del aceite usado al remplazarse al $30 \%$ del crudo residual del horno cementero, se observó que su poder calorífico está cerca de 9.300 $\mathrm{kcal} / \mathrm{kg}$ de clínker, siendo un balance positivo. La tecnología que cuenta el quemador principal del horno cementero, demostró que es apto para la destrucción controlada y efectiva de los aceites usados, además de segura por todas las condiciones por su encapsulamiento en la estructura interna del clínker que posee este proceso. La propuesta de re-uso del aceite usado generó alternativas de disposición final y aprovechamiento energético para la industria cementera, se verá reflejado en el crecimiento económico del cantón Riobamba a la medida que contribuya en la reducción de este tipo de desechos peligrosos y mantenga una sustentabilidad ambiental acorde a generar mayor valor agregado.

- En la fase experimental de la investigación se caracterizó varias mezclas llegando a una propuesta óptima entre el $70 \%$ de residuo petrolero y $30 \%$ de aceite usado; el tratamiento estadístico demuestra que no existen diferencias significativas en sus componentes, es decir es apto a un proceso de combustión del horno cementero. Técnicamente, la inyección de aceite usado proveniente de fuentes automotrices, genera un impacto en la capacidad de producción de plantas cementeras con un ahorro anual energético anual por tonelada de clínker producida, cumpliéndose la reducción del costo especifico de la energía térmica. La valorización energética aceites usados ante un coprocesamiento, mostró una alternativa de gestión energética basada en un 
programa de control ambiental ante su disposición final, mostrando ser secundarios a los beneficios establecidos por la recuperación.

- Los aceites lubricantes usados generados por sector industrial y fuentes automotrices de la ciudad de Riobamba constituyen residuos potenciales para su aprovechamiento energético ya que contienen una elevada capacidad calorífica, pudiéndose sustituir a procesos con tecnología de mezcla con el combustible en un sistema térmico de un horno de cemento, cumpliéndose con requisitos de calidad y manejo seguro en sus procesos de combustión a medida que este tipo de desecho peligroso genere alternativa de disposición final y contribuya en la reducción alcanzando soluciones de mejoramiento del impacto ambiental.

- De acuerdo a los requerimientos de combustibles mostrados en la tabla 1, las mezclas evaluadas poseen elevado poder calorífico, superior a $9300 \mathrm{Kcal} / \mathrm{kg}$; según los requisitos de combustibles de la norma ASTM D 6448, se observa, la mezcla de prueba P8, tiene mayor contenido energético cerca de $9566 \mathrm{kcal} / \mathrm{kg}$ que el especificado para el grado RFO4 calidad que evalúa al combustible fabricado en su totalidad o en parte con aceite lubricante usado; durante la fase experimental se demuestra que la potencialidad del aceite usado al remplazarse al $60 \%$ de combustible industrial en un sistema térmico de un horno cementero, es el más óptimo ya que estadísticamente no existen diferencias significativas en sus componentes siendo acto a un proceso de combustión de equipos industriales que queman combustibles en diversas condiciones climáticas y de operación.

\section{Referencias Bibliográficas.}

- Abdelghani, L., Denidni, T., and Nedil, M. (2012a). Design of a new Ultra-wideband $4 \times 4$ Butler matrix for beamforming antenna applications. Paper presented at Antennas and Propagation Society International Symposium (APSURSI), Retrieved from https://ieeexplore.ieee.org/document/6348774/.

- Abdelghani, L., Denidni, T., and Nedil, M. (2012b). Ultra-broadband $4 \times 4$ compact Butler matrix using multilayer directional couplers and phase shifters. Paper presented at Microwave Symposium Digest (MTT), Retrieved from https://ieeexplore.ieee.org/document/861760/.

- Balanis, C. A. (2005). Antenna Theory, Analysis and Design, third edition. New Jersey: John Wiley \& Sons.

- Bialkowski, M., Tsai, F., Su, Y., and Cheng, K. (2008). Design of fully integrated $4 \times 4$ and 8x8 Butler matrices in microstrip/slot technology for ultra wideband smart antennas. Paper presented at Antennas and Propagation Society International Symposium, IEEE, Retrieved from https://ieeexplore.ieee.org/document/4619182/.

- Boole, G. (1857). On the comparasion of transcendents with certain applications to the theory of definite integrals. Philosophical Transactions of the Royal Society of London, 147(III), 745-803.

- Moscoso-Martir, A., Molina-Fern’ andez, I., and Ortega-' Monux, A. (2014). Wideband slot-coupled butler ma- trix. IEEE Microwave and Wireless Components Letters, 24(12), 848-850. 
- Musa, L. and Smith, M. (1986). Microstrip rotman lens port design. Paper Presented at Antennas and Propagation Society International Symposium, Retrieved from https://ieeexplore.ieee.org/document/1149578/.

- Musa, L. and Smith, M. (1989). Microstrip port design and sidewall absorption for printed Rotman lenses. Paper presented at IEE Proceedings H - Microwaves, Antennas and Propagation, Retrieved from https://ieeexplore.ieee.org/document/16619/.

- Walpole , R. E., Myers, R. H., \& Myers, S. L. (1999). Probabilidad y estadística para ingenieros. Mexico: Hall Hispanoamerica.

- Texto Unificado de Legislación Secundaria. (2015). Texto Unificado de Legislación Secundaria. En D. P. Hugo, Reforma del libro VI de Texto Unificado de Legislación Secundaria (págs. 3-53). Quito. 


\section{PARA CITAR EL ARTÍCULO INDEXADO.}

Albuja Echeverría, R., López Ayala, J., Guilcapi Mosquera, J., \& Guamán Reinoso, C. (2019). Valor energético del aceite usado en sistemas de combustión térmicos de la industria cementera del país. Ciencia Digital, 3(4.2), 100-115. https://doi.org/10.33262/cienciadigital.v3i4.2.1012

El artículo que se publica es de exclusiva responsabilidad de los autores y no necesariamente reflejan el pensamiento de la Revista Ciencia Digital.

El artículo queda en propiedad de la revista y, por tanto, su publicación parcial y/o total en otro medio tiene que ser autorizado por el director de la Revista Ciencia Digital.
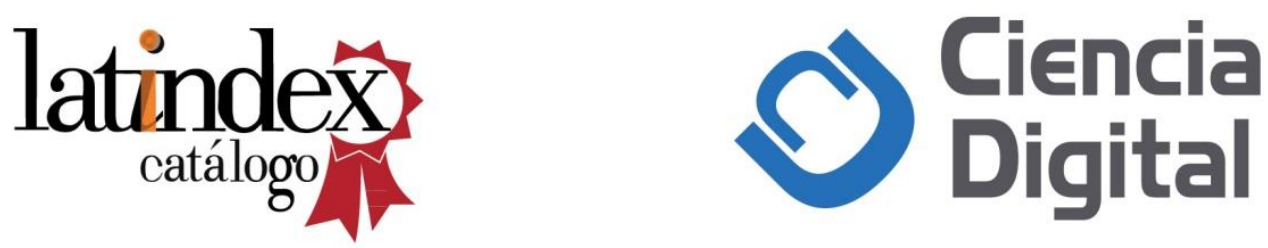